\title{
COMPORTAMENTO DE LEVEDURAS DO GÊNERO CANDIDA "IN VITRO" A ANTIFÚNGICOS
}

\author{
Maria do Socorro de Sousa FURTADO', Débora da Rocha PIMENTA², \\ Jeronilson de Almeida FERREIRA', Ana Cláudia CORTÊ $\mathbf{Z}^{1}$
}

RESUMO - Estudou-se o comportamento de leveduras do gênero Candida a antifúngicos, pela determinação da Concentração Inibitória Mínima (CIM) e da Concentração Fungicida Mínima (CFM) "in vitro"de 30 cepas de Candida frente aos antifúngicos: miconazol, cetoconazol e anfotericina B. Empregou-se o método de diluição em meio líquido e os antifüngicos foram diluidos visando proporcionar concentrações a partir de 0,06 a $128 \mu \mathrm{g} / \mathrm{mL}$. O inóculo foi padronizado ajustando-se a suspensão para conter $1 \times 10^{6} \mathrm{ufc} / \mathrm{mL}$. A concentração para a qual houve maior convergência de cepas foi de $1 \mu \mathrm{g} / \mathrm{mL}(26,5 \%)$ para anfotericina B. Para miconazol foi de $16 \mu \mathrm{g} / \mathrm{mL}(26,0 \%)$ e para cetoconazol $32 \mu \mathrm{g} / \mathrm{mL}(23,0 \%)$. Os valores de CFM foram de $2 \mu \mathrm{g} / \mathrm{mL}(23,5 \%)$ para anfotericina $\mathrm{B}$, de 16 e $64 \mu \mathrm{g} / \mathrm{mL}(26,5 \%)$ para miconazol e 32 e $64 \mu \mathrm{g} / \mathrm{mL}(30,0 \%)$ para cetoconazol. Este imidazólico mostrou os valores de CIM e CFM mais elevados atingindo até $128 \mu \mathrm{g} / \mathrm{mL}$ em algumas espécies. As espécies de Candida mostraram-se mais sensiveis à Anfotericina B, quando comparadas em relação aos antifúngicos testados. Sobre o desempenho das espécies de Candida, melhor padrão de comportamento foi verificado para $C$, albicans com niveis mais baixos de sensibilidade.

Palavras-chave: Candida; antifúngicos

\section{Sensitivity of Candida Yeasts to Antifungals "In Vitro"}

ABSTRACT - The response of Candida species to antifungal agents was evaluated by determination of Minimum Inhibitory Concentration (MIC) and Minimum Fungicidal Concentration (MFC) "in vitro". Amphotericin B, miconazole and ketoconazole were tested at concentrations between 0,06 to $128 \mu \mathrm{g} / \mathrm{mL}$ using the broth dilution method. Inocula of 30 Candida strains were standardized by adjusting the suspension to contain $1 \times 10^{6} \mathrm{cfu} / \mathrm{mL}$. Highest strain convergence was seen at MIC of $1,0 \mu \mathrm{g} / \mathrm{mL}$ for amphotericin B $(26,5 \%), 16 \mu \mathrm{g} / \mathrm{mL}$ for miconazole $(26,0 \%)$ and $32 \mu \mathrm{g} /$ $\mathrm{mL}$ for ketoconazole $(23,0 \%)$. MFC values were $2 \mu \mathrm{g} / \mathrm{mL}$ for amphotericin B $(23,5 \%), 16$ and $64 \mu \mathrm{g} /$ $\mathrm{mL}$ for miconazole $(26,5 \%)$ and 32 and $64 \mu \mathrm{g} / \mathrm{mL}$ for ketoconazole $(30 \%)$. This imidazole showed the highest MIC and MFC with values up to $128 \mu \mathrm{g} / \mathrm{mL}$. All Candida species, were more sensitive to amphotericin B than to the others two drugs, and C. albicans was the species with the highest sensitivity.

Key-words: Candida, antifungal

\section{INTRODUÇÃO}

As leveduroses estão entre as infecções mais comuns causadas por fungos. O gênero Candida vive saprofiticamente no tubo digestivo do homem e de certos animais, podendo ter ação patogênica em quase todas as partes do organismo humano. As manifestações clínicas da doença são as mais variadas, podendo ser subaguda, aguda ou crônica
O envolvimento pode ser localizado na boca, orofaringe, couro cabeludo, vagina, unhas, brônquios, pulmões, trato gastrointestinal ou generalizado como septicemia, endocardite e meningite (ARENAS, 1993).

Os processos patológicos tembém são variados indo desde irritação e inflamação até uma resposta granulomatosa e supurativa.

As leveduras do gênero Candida, comportam-se como agentes oportunistas.

\footnotetext{
Instituto Nacional de Pesquisas da Amazonia, Caixa Postal 478, 69011-970,Manaus, Amazonas, Brasil

2 Bolsista de Iniciação Científica (PIBIC-INPA)
} 
De acordo com observações realizadas (LACAZ, 1980; MALLIE et al., 1982) sua virulência está condicionada a fatores ligados ao hospedeiro, o qual em condições fisiológicas ou na vigência de entidades mórbidas diversas, ou face à iatrofarmacogenia desenvolve quadros clínicos variados, com lesões superficiais ou profundas.

Nos pacientes idosos, debilitados, recém-nascidos prematuros, e, desnutridos como também em pacientes infectados pelo HIV, LACAZ (1985) faz referência à ocorrência de candidíase, em suas variadas formas clínicas.

Com relação aos fatores locais (LACAZ, 1980; LACAZ et al., 1984) referem como um dos principais, a umidade. Assim, trabalhadores em bares e lavadeiras, estando permanentemente em contato com água, muitas vêzes contaminada, e sabões, possibilitam a colonização do fungo, principalmente em sulcos ou dobras cutâneas onde as leveduras encontram melhores condições para seu crescimento. A maceração da pele por fatores mecânicos ou quimicos, também favorece o crescimento da levedura, sob a forma filamentosa.

Trabalhos anteriores (MC GINNIS, 1980) têm demonstrado que o gênero Candida apesar de seu caráter saprofitico, pode desenvolver grave patogenicidade na ocorrência de uma mudança em seu ambiente natural como por exemplo em decorrência de alterações do $\mathrm{pH}$, aumento de temperatura e umidade entre outros fatores. Quando isto acontece, estabelece-se um desequilibrio na sua população que passa a desenvolver-se em grande quantidade e devido ao seu caráter oportunista, tornar-se também um patógeno.

Em observação às citações de IWATA \& YAMAGUCHI (1973), FROMTLING \& SHADOMY (1983), MINAGAWA et al. (1983), GUGLIELMINETTI \& CREMA (1984), GERACI (1986), HOEPRICH \& MERRY (1986) e ODDS et al. (1986), devido ao grande índice de micoses ocasionadas por estas leveduras e com o crescimento do número de portadores de HIV que estão atualmente entre os grupos mais susceptiveis a estas infecções, torna-se necessário o desenvolvimento de testes de sensibilidade para analisar o comportamento das espécies do gênero mediante quimioterápicos para estabelecer o diagnóstico e a terapêutica adequados, visto que na maioria dos casos poderá tornar-se generalizada e resistente aos antifúngicos usuais .

\section{MATERIAL E MÉTODOS}

Foram utilizadas 30 cepas de leveduras isoladas de material biológico humano de pacientes com suspeita clinica de dermatomicose. O material examinado, constou de escamas epidérmicas, fragmentos de unha e secreções (Tab. 1).

Realizou-se Testes de sensibilidade a antifúngicos pela determinação da Concentração Inibitória Mínima (CIM) e Concentração Fungicida Mínima (CFM).

Os agentes antifúngicos empregados nos testes de sensibilidade foram os derivados imidazólicos: cetoconazol (Johnson \& Johnson Indústria e Comércio S/A), miconazol (Johnson \& Johnson Indústria e Comércio S/A) e o poliênico anfotericina B (Squibb Indústria Química $\mathrm{S} / \mathrm{A}$ ), na forma micropulverizada.

O método utilizado nos experimentos foi o de diluição em meio líquido, sendo 
Tabela 1. Origem das leveduras do gênero Candida, por localização.

Gênero/Espécie

\begin{tabular}{|c|c|c|c|c|c|}
\hline Local & C. albicans & C. guilliermondii & C. parapsilopsis & C. stellatoideae & Total \\
\hline Unha & 13 & 2 & 1 & 1 & 17 \\
\hline Glande & 4 & - & 1 & - & 5 \\
\hline Mão & 2 & - & - & - & 2 \\
\hline Axila & 2 & - & - & - & 2 \\
\hline Escarro & 1 & - & . & - & 1 \\
\hline Pé & 1 & - & - & - & 1 \\
\hline Inguinal & 1 & - & - & - & 1 \\
\hline Glúteo & 1 & - & - & - & 1 \\
\hline Total & 25 & 2 & 2 & 1 & 30 \\
\hline
\end{tabular}

$C=$ Candida

empregado o meio Yeast Nitrogen Base (DIFCO) $\mathrm{pH} 6,0$. Os antifúngicos foram dissolvidos em $5,0 \mathrm{~mL}$ de dimetil sulfóxido (DMSO), para obter soluções contendo $10.000 \mu \mathrm{g} / \mathrm{mL}$. Essas soluções foram acondicionadas em alíquotas de $1 \mathrm{~mL}$ em tubos rosqueáveis e estocadas a $70^{\circ} \mathrm{C}$. Para preparar solução de trabalho $(1000 \mu \mathrm{g} / \mathrm{mL})$, o estoque foi diluído (1:100) em meio líquido, conservado a $70^{\circ} \mathrm{C}$ e usado por um período de até 15 dias. A diluição seriada $(1: 2)$ foi padronizada para 12 concentrações diferentes, de $128 \mu \mathrm{g} / \mathrm{mL}$ a $0,06 \mu \mathrm{g} / \mathrm{mL}$, para obter $5 \mathrm{~mL}$ de meio com cada concentração final dos agentes antifúngicos. Para inoculação, utilizou-se cepas recém-cultivadas (48 horas) em meio de Sabouraud Agar.

Uma suspensão foi preparada da porção superficial da cultura em água destilada estéril. Desta suspensão, obtevese por contagem em hematocitômetro, uma quantidade padrăo de células leveduriformes $\left(1 \times 10^{6} \mathrm{ufc} / \mathrm{mL}\right)$ que foi usada para inocular $100 \mu \mathrm{L}$ para cada tubo de ensaio. Estes testes foram incubados a $30^{\circ} \mathrm{C}$ e interpretados após 48 horas.
Sob condições padronizadas, a CIM foi considerada como a menor concentração que inibiu crescimento fúngico. Leitura positiva nos tubos, foi aquela em que crescimento foi possível de ser observado à vista desarmada. Para a determinação da CFM, os tubos com ausência de crescimento foram reinoculados em tubos de ensaio contendo novo meio sem o agente antifúngico. Esta nova série foi incubada a $30^{\circ} \mathrm{C}$ e lida após 48 horas.

\section{RESULTADOS E DISCUSSÃO}

As 30 cepas de Candida testadas com relação ao seu comportamento frente aos derivados imidazólicos cetoconazol, miconazol e o poliênico anfotericina $\mathrm{B}$, mostraram-se sensíveis aos mesmos em gráus variáveis, o que se observa pelos valores de CIM e de CFM encontrados. Com relação a anfotericina $\mathrm{B}$, os valores de CIM encontrados foram de $0,12 \mu \mathrm{g} / \mathrm{mL}$ a $4 \mu \mathrm{g} / \mathrm{mL}$ para $C$, albicans; de 0,25 a 2 $\mu \mathrm{g} / \mathrm{mL}$ para C. guilliermondiip, de 0,12 a $2 \mu \mathrm{g} / \mathrm{mL}$ para C. parapsilopsis e de $4 \mu \mathrm{g} / \mathrm{mL}$ para C. stellatoideae. Os valores 
de CFM foram de 0,2 a $64 \mu \mathrm{g} / \mathrm{mL}$ para $C$. albicans, de 1 a $4 \mu \mathrm{g} / \mathrm{mL}$ para $C$. guilliermondii; de 1 a $8 \mu \mathrm{g} / \mathrm{mL}$ para $C$. parapsilopsis e de $64 \mu \mathrm{g} / \mathrm{ml}$ para $C$. stellatoideae (Tab. 2).

Os níveis de CIM encontrados com cetoconazol foram para $C$. albicans,
$(26,5 \%)$ das cepas na CIM e para a de $2 \mu \mathrm{g} / \mathrm{mL}$ na CFM, correspondendo a 7 $(23,5 \%)$ das cepas, enquanto que para o cetoconazol maior concentração se situou em $32 \mu \mathrm{g} / \mathrm{mL}$ com 7 ( $23 \%)$ das cepas na CIM e entre as de $32 \mu \mathrm{g} /$ $\mathrm{mL}$ a $64 \mu \mathrm{g} / \mathrm{mL}$ na CFM com $9(30 \%)$

Tabela 2. Atividade antifúngica de Anfotericina B frente ao gênero Candida pela CIM e CFM.

\begin{tabular}{lcrr}
\hline Espécies & № Cepas & CIM & CFM \\
\hline C. albicans & 25 & $0,12 \mathrm{a} 4 \mu \mathrm{g} / \mathrm{mL}$ & $0,25 \mathrm{a} 64 \mu \mathrm{g} / \mathrm{mL}$ \\
C. guilliermondii & 2 & $0,25 \mathrm{a} 2 \mu \mathrm{g} / \mathrm{mL}$ & 1 a $4 \mu \mathrm{g} / \mathrm{mL}$ \\
C. parapsilopsis & 2 & $0,12 \mathrm{a} 2 \mu \mathrm{g} / \mathrm{mL}$ & 1 a $8 \mu \mathrm{g} / \mathrm{mL}$ \\
C. stellatoideae & 1 & $4 \mu \mathrm{g} / \mathrm{mL}$ & $64 \mu \mathrm{g} / \mathrm{mL}$ \\
\hline
\end{tabular}

$C=$ Candid $a$

de 0,25 a $64 \mu \mathrm{g} / \mathrm{mL}$; para $C$. das cepas; para miconazol, houve guilliermondii de 0,5 a $4 \mu \mathrm{g} / \mathrm{mL}$; para $C$. parapsilopsis de 2 a $8 \mu \mathrm{g} / \mathrm{mL}$ e para C. stellatoideae $1 \mu \mathrm{g} / \mathrm{mL}$. Os valores de CFM, foram para C. albicans de 2 a 128 $\mu \mathrm{g} / \mathrm{mL}$; para C. guilliermondii de 8 a $32 \mu \mathrm{g} /$ $\mathrm{mL}$; para C. parapsilopsis de 4 a $32 \mu \mathrm{g} /$ $\mathrm{mL}$ e para C. stellatoideae de $8 \mu \mathrm{g} / \mathrm{mL}$.

Os niveis de CIM encontrados para miconazol foram para C. albicans de 0,12 a $32 \mu \mathrm{g} / \mathrm{mL}$, para $C$. guilliermondii de $8 \mu \mathrm{g} / \mathrm{mL}$, para $C$. parapsilopsis 4 a $16 \mu \mathrm{g} / \mathrm{mL}$ e para $C$. stellatoideae, de $16 \mu \mathrm{g} / \mathrm{mL}$ (Tab. 4)

Os valores de CFM encontrados foram de 1 a $128 \mu \mathrm{g} / \mathrm{mL}$ para $C$. albicans, de 8 a $16 \mu \mathrm{g} / \mathrm{mL}$ para $C$. guilliermondii, de $16 \mu \mathrm{g} / \mathrm{mL}$ para $C$. parapsilopsis e $16 \mu \mathrm{g} / \mathrm{mL}$ para $C$. stellatoideae (Tab. 6).

Quanto ao gráu de convergência para uma determinada concentração, observamos que em relação a anfotericina $\mathrm{B}$, houve maior direcionamento das cepas para a de $1 \mu \mathrm{g} / \mathrm{mL}$ correspondendo a 8 maior convergência para a concentração de $16 \mu \mathrm{g} / \mathrm{mL}$ com 8 $(26,5 \%)$ das cepas na CIM e de $16 \mathrm{e}$ $64 \mu \mathrm{g} / \mathrm{mL}$ com $8(26,5 \%)$ das cepas na CFM (Tabs. 3, 5 e 7).

De um modo geral, as cepas de Candida examinadas, mostraram-se mais sensíveis ao poliênico anfotericina

Tabela 3. Niveis de sensibilidade (CIM e CFM \%) de Candida para Anfotericina B.

\begin{tabular}{ccc}
\hline & \multicolumn{2}{c}{ Total de cepas } \\
\cline { 2 - 3 } Concentrações $(\mu \mathrm{g} / \mathrm{mL})$ & CIM $\%$ & \multicolumn{1}{c}{ CFM $\%$} \\
\hline 0,06 & - & - \\
0,12 & $6(20,0 \%)$ & - \\
0,25 & $4(13,5 \%)$ & $2(6,5 \%)$ \\
0,5 & $1(3,5 \%)$ & $1(3,5 \%)$ \\
1 & $8(26,5 \%)$ & $3(10,0 \%)$ \\
2 & $6(20,0 \%)$ & $7(23,5 \%)$ \\
4 & $5(16,5 \%)$ & $3(10,0 \%)$ \\
8 & - & $5(16,5 \%)$ \\
16 & - & $2(6,5 \%)$ \\
32 & - & $2(6,5 \%)$ \\
64 & - & $5(16,5 \%)$ \\
128 & - & - \\
\hline Total & $30(100 \%)$ & $30(100 \%)$ \\
\hline
\end{tabular}


Tabela 4. Atividade antifúngica do Cetoconazo] frente ao gênero Candida pela CIM e CFM.

\begin{tabular}{lrr}
\hline Espécies & \multicolumn{1}{c}{ CIM } & \multicolumn{1}{c}{ CFM } \\
\hline Calbicans & $0,25 \mathrm{a} 64 \mu \mathrm{g} / \mathrm{mL}$ & $2 \mathrm{a} 128 \mu \mathrm{g} / \mathrm{mL}$ \\
C. guilliermondii & $0,5 \mathrm{a} 4 \mu \mathrm{g} / \mathrm{mL}$ & $8, \mathrm{a} 32 \mu \mathrm{g} / \mathrm{mL}$ \\
C. parapsilopsis & $2 \mathrm{a} 8 \mu \mathrm{g} / \mathrm{mL}$ & $4 \mathrm{a} 32 \mu \mathrm{g} / \mathrm{mL}$ \\
C. stellatoideae & $1 \mu \mathrm{g} / \mathrm{mL}$ & $8 \mu \mathrm{g} / \mathrm{mL}$ \\
C $=$ Candida & & \\
\hline
\end{tabular}

Tabela 5. Niveis de sensibilidade (CIM e CFM \%) de Candida para Cetoconazol.

\begin{tabular}{ccc}
\hline & \multicolumn{2}{c}{ Total de cepas } \\
\cline { 2 - 3 } Concentrações $(\mu \mathrm{g} / \mathrm{mL})$ & \multicolumn{1}{c}{ CIM $\%$} & CFM $\%$ \\
\hline 0,06 & - & - \\
0,12 & $1(3,5 \%)$ & - \\
0,25 & $4(13,5 \%)$ & - \\
0,5 & $2(6,5 \%)$ & - \\
1 & $4(13,5 \%)$ & $1(3,5 \%)$ \\
2 & $3(10,0 \%)$ & $2(6,5 \%)$ \\
4 & $3(10,0 \%)$ & $1(13,5 \%)$ \\
8 & $1(3,5 \%)$ & $2(6,5 \%)$ \\
16 & $7(23,0 \%)$ & $9(30,0 \%)$ \\
32 & $5(16,5 \%)$ & $9(30,0 \%)$ \\
64 & - & $3(10,0 \%)$ \\
128 & $30(100 \%)$ & $30(100 \%)$ \\
\hline Total & &
\end{tabular}

B tanto com relação a CIM quanto com a CFM o que se constata pelo maior número de cepas localizadas em baixas concentrações (Tab. 3).

Entre os dois imidazólicos testados, o miconazol foi o antifúngico para o qual as cepas mostraram melhor desempenho com niveis mais baixos de sensibilidade, enquanto que para o cetoconazol, os níveis de sensibilidade foram um pouco mais elevados (Tabs. 5 e 7 ).

Apesar de neste estudo termos testado 25 cepas de $C$. albicans contra 5 cepas de Candida não albicans, comparando-se o desempenho das espécies de Candida com relação aos antifúngicos, verificamos melhor padrão de comportamento de $C$. albicans com niveis mais baixos de sensibilidade; entretanto estes resultados não são conclusivos e não válidos como referencial para as diversas espécies de Candida. Os níveis de sensibilidade encontrados, são demonstrados pela CIM (Tabs. 2 , 4 e 6 ).

Tabela 6. Atividade antifúngica do Miconazol frente ao gênero Candida pela CIM e CFM.

\begin{tabular}{lrc}
\hline Espécies & \multicolumn{1}{c}{ CM } & \multicolumn{1}{c}{ CFM } \\
\hline C.albicans & $0,12 \mathrm{a} 32 \mu \mathrm{g} / \mathrm{mL}$ & 1 a $128 \mu \mathrm{g} / \mathrm{mL}$ \\
C.guilliermondii & $8 \mu \mathrm{g} / \mathrm{mL}$ & 8, a $16 \mu \mathrm{g} / \mathrm{mL}$ \\
C.parapsilopsis & $4 \mathrm{a} 16 \mu \mathrm{g} / \mathrm{mL}$ & $16 \mu \mathrm{g} / \mathrm{mL}$ \\
C.stellatoideae & $16 \mu \mathrm{g} / \mathrm{mL}$ & $16 \mu \mathrm{g} / \mathrm{mL}$ \\
C=Candida & & \\
\hline
\end{tabular}

Tabela 7. Niveis de sensibilidade (CIM e CFM \%) de Candida para Miconazol.

\begin{tabular}{ccc}
\hline & \multicolumn{2}{c}{ Total de cepas } \\
\cline { 2 - 3 } Concentrações $(\mu \mathrm{g} / \mathrm{mL})$ & CIM $\%$ & \multicolumn{1}{c}{ CFM \% } \\
\hline 0,06 & $1(3,5 \%)$ & - \\
0,12 & - & - \\
0,25 & $2(6,5 \%)$ & - \\
0,5 & - & $1(3,5 \%)$ \\
1 & $5(16,5 \%)$ & - \\
2 & $4(13,5 \%)$ & $2(6,5 \%)$ \\
4 & $4(13,5 \%)$ & $4(13,5 \%)$ \\
8 & $8(26,5 \%)$ & $8(26,5 \%)$ \\
16 & $6(20,0 \%)$ & $6(20,0 \%)$ \\
32 & - & $8(26,5 \%)$ \\
64 & - & $1(3,5 \%)$ \\
128 & $30(100 \%)$ & $30(100 \%)$ \\
\hline Total & &
\end{tabular}

\section{Bibliografia Citada}

ARENAS, R. 1993. Micologia Médica Ilustrada. Ed. Interamericana. Mc Grow Hill, México. 397 p.

FROMTLING, R. A; YU, H. P; SHADOMY, S. 1983. In vitro inhibitory activities of 2 new orally absorbable imidazole derivatives : Bay 7133 and Bay 19139 . Sabouraudia, 21; 179-184

GERACI, P. 1986. Miconazole in the tropical treatments vulvovaginitis caused by 
Candida albicans. Gazz. Med. Italarch Sci. Med., 145 (3): 129-132

GUGLIELMINETTI, M; CREMA, F. 1984. Sensibilitá dei lieviti e dei funghi filamentosi gli antifungini. Studio comparativo in vitro. Farmaco Edizione Pratica, 39(5): 139- 47

HOEPRICH,P. D.; MERRY, J. M. 1986. Influence of culture medium on susceptibility testing with Bay 7133 and Ketoconazole J.Clin.Microbiol., 24(2): 269-71

IWATA, K.; IAMAGUCHI, T. 1973. Mode of action of Clotrimazole. Sabouraudia, 11: $58-65$

LACAZ, C. S. 1980. Candidiases. Ed. Pedagógica e Universitária Ltda., São Paulo, $190 \mathrm{p}$.

1985. AIDS, Doutrinas, Aspectos Iatrofilosóficos e Infecções Oportunistas Associadas. Sarvier, S.Paulo, 124 p ; PORTO, E; MARTINS, J. E. C., 1984. Micologia Médica, $8^{\text {a }}$ ed, S.Paulo, Sarvier, $479 \mathrm{p}$.
MALLIE, M.; JOUVERT, S.; LEBECQ, J. C. ; BASTIDE, J. M. 1982. Valeur comparee de diferents methodes d'evaluation de la C.M.I. des antifongiques: sensibilité de Candida albicans a l'econazole. Societé Française de Mycologia Medicale, 155-60

MINAGAWA, H.; KITAURA, K.; NAKAMIZO, N. 1983. Effects of $\mathrm{pH}$ on the activity of Ketoconazole against Candida albicans . Antimicrobiol. Agents and Chemother., 23(1): 107-07

Mc GINNIS, M.R. 1980, Laboratory handbook of Medical Micology. New York Academic Press, 411-31

ODDS, F. C.; ABOTT, A. B.; PYE, G.; TROKE, P. F. 1986. Improved method for estimation of azole antifungal inhibitory concentrations against Candida species, based on azole antibiotic interactions. J. Med. and Veter: Mycol., 24: 305-311 